\title{
MULTIPLICATION RINGS AS RINGS IN WHICH IDEALS WITH PRIME RADICAL ARE PRIMARY
}

\author{
ROBERT W. GILMER, JR. ${ }^{\text {BY }}{ }^{\text {B }}$ AND JOE LEONARD MOTT
}

A commutative ring $R$ is called an $A M$-ring (for allgemeine multiplikationsring) if whenever $A$ and $B$ are ideals of $R$ with $A$ properly contained in $B$, then there is an ideal $C$ of $R$ such that $A=B C$. An $A M$-ring $R$ in which $R A=A$ for each ideal $A$ of $R$ is called a multiplication ring. Krull introduced the notion of a multiplication ring in [11], [13]. Akizuki is responsible for the more general concept of an $A M$-ring in [1], but Mori has developed most of the structure theory for such rings in $[14],[15],[16],[17]$, and [18].

An important property of an $A M$-ring $R$ is that $R$ satisfies what Gilmer called condition $\left({ }^{*}\right)$ in $[7]$ and $[8]:$ An ideal of $R$ with prime radical is primary. In $\S 1$, new results concerning rings in which $\left(^{*}\right)$ holds are given. These are applied to obtain structure theorems for $A M$-rings in $\S 2$.

In [10, p. 737], Krull introduced the notion of the kernel of an ideal $A$ in a commutative ring $R$, which is defined thusly: if $\left\{P_{\alpha}\right\}$ is the collection of minimal prime ideals of $A$, then by an isolated primary component of $A$ belonging to $P_{\alpha}$ we mean the intersection $Q_{\alpha}$ of all $P_{\alpha}$-primary ideals which contain $A$. The kernel of $A$ is the intersection of all $Q_{\alpha}^{\prime}$ s. Mori considered rings in which every ideal is equal to its kernel in [16] and [17]. In $\S 1$, it is shown that a ring $R$ satisfies $\left(^{*}\right)$ if and only if every ideal of $R$ is equal to its kernel.

In $\S 2$ of this paper, we consider rings $R$ satisfying condition (F): If $A$ and $P$ are ideals of $R$ such that $P$ is prime and $A$ is properly contained in $P$, there is an ideal $B$ such that $A=P B$. Theorems 12 and 13 show that such a ring is an $A M$-ring. This generalizes a result proved by Mott in [19] for rings with unit. This theorem might be compared with the result of Cohen $[3$, Theorem 2, p. 29], that a ring in which every prime ideal is finitely generated is a Noetherian ring and to the theorem of Nakano in [20, p. 234] which states that every nonzero ideal of an integral domain $D$ with unit is invertible provided every nonzero prime ideal of $D$ is invertible. In addition, several new sets of necessary and sufficient conditions that a ring $R$ be an $A M$-ring are given in $\S 2$. An equally significant aspect of $\S 2$ in our eyes is that in the process of proving Theorem 12, many of the known results concerning $A M$-rings are proved in a way we feel is clearer and more straight-

Received by the editors July 1, 1963.

( ${ }^{1}$ This author received support for this research from the Office of Naval Research under contract number NONR (G) 00099-62. 
forward than proofs already in the literature. The remaining known structure theorems concerning $A M$-rings are then proved in Theorems $16-17$ by using the results leading up to the proof of Theorem 12.

1. Rings in which condition $\left({ }^{*}\right)$ holds. This section extends results of Gilmer in [7] and [8] concerning rings in which every ideal with prime radical is primary, the so-called rings satisfying condition $\left(^{*}\right)$. Throughout this paper we shall use extensively certain results of the papers just cited. For sake of reference, we shall number these, but first we need two definitions.

Definitions. A ring $R$ is called a primary ring if $R$ contains at most two prime ideals [5]. A primary domain is a primary ring without proper divisors of zero. A ring $S$ is called a $u$-ring if the only ideal $A$ of $S$ such that $\sqrt{ } A=S$ is $S$ itself.

Theorem 1. A ring $R$ satisfies $\left(^{*}\right)$ if and only if $R$ is one of the following:

(a) a primary domain,

(b) a ring, every element of which is nilpotent,

(c) a zero-dimensional u-ring, or

(d) a one-dimensional u-ring in which each nonmaximal prime ideal $P$ of $R$ has the property that if $M$ is a maximal ideal such that $P<M<R$, and if $p \in P$, then $p \in p M$.

In Theorem $1,(d)$ is equivalent to

(d') $R$ is a one-dimensional $u$-ring such that $P_{M}=(0)$ if $P$ and $M$ are prime ideals of $R$ with $P<M<R$. (If $A$ and $B$ are prime ideals of a ring $R$ with $A \subset B, A_{B}$ will denote the extension of the ideal $A$ to the ring $R_{B}$ $[21$, p. 218]), or

$\left(\mathrm{d}^{\prime \prime}\right) R$ is a one-dimensional $u$-ring and if $P$ is a nonmaximal prime ideal of $R$, then $\sqrt{ } A=P$ implies $A=P$.

The proof of Theorem 1 is in [7], as is the proof of

Theorem 2. Suppose $R$ is a u-ring. Then

(a) $R$ is idempotent and every proper ideal of $R$ is contained in a proper prime ideal.

(b) If $M$ is a maximal ideal of $R, M$ is prime and $R / M$ is a field.

(c) If $R$ is one-dimensional and an integral domain, $R$ contains an identity.

Theorem 3, which follows, represents a broad generalization of Theorem 2(c). To prove Theorem 3 we need two lemmas.

Leмма 1. Let $R$ be a commutative ring with identity and let $P$ be a nonzero proper prime ideal of $R$. If $P$, considered as a ring, is a u-ring and the set of prime ideals not containing $P$ is inductive, then $P$ is comaximal with every prime ideal not containing it, and $R_{P}$ is a field.

Proof. We suppose there is a prime ideal which does not contain $P$ and 
which is not comaximal with $P$. By Zorn's lemma we may find a prime, $P^{\prime}$, which is maximal with respect to these two properties. Since $P+P^{\prime}$ is proper, we may find a prime $Q \supset P+P^{\prime}$, which is proper and minimal over $P+P^{\prime}$. Our constructions guarantee that $Q$ is also minimal over $P^{\prime}$. Now in $R_{Q}, P_{Q} \nsubseteq P_{Q}^{\prime}$ so we choose $\Pi \in P_{Q}-P_{Q}^{\prime}$. Because $Q_{Q}$ is a minimal prime over $P_{Q}^{\prime}, P_{Q}^{\prime}+(\Pi)$ is $Q_{Q}^{\prime}$-primary.

We next note that $P_{Q}$ is also a $u$-ring, so that $P_{Q}=P_{Q}\left[P_{Q}^{\prime}+(\Pi)\right]$ since this latter ideal has radical $P_{Q}$. Hence $P_{Q} \subset P_{Q}^{\prime}+(\Pi)$. Thus $P_{Q}=P_{Q}^{2} \subset$ $P_{Q} P_{Q}^{\prime}+P_{Q} \Pi \subset P_{Q}$ and $P_{Q}=P_{Q} P_{Q}^{\prime}+(\Pi)$. Similarly, $P_{Q}=P_{Q} P_{Q}^{\prime}+\Pi^{2} R_{Q}$. Hence $\Pi=\sigma+\Pi^{2} \lambda$ where $\sigma \in P_{Q} P_{Q}^{\prime}$. Thus $\Pi(1-\lambda \Pi) \in P_{Q} P_{Q}^{\prime} \subset P_{Q}^{\prime}$. But $1-\lambda \Pi$ is a unit so $\Pi \in P_{Q}^{\prime}$ and we thus arrive at a contradiction. The rest of the lemma is immediate.

Lemma 2. If $R$ and $P$ are as in Lemma 1 and, in addition, $R / P$ satisfies the descending chain condition on its prime ideals, then, for all $\Pi \in P, \Pi \in \Pi P$.

Proof. We claim first that $P_{Q}=0$ for all prime ideals, $Q$, such that $P \subset Q$. If not, by the chain condition, pick $Q$ minimal with respect to the properties that $P \subset Q$ and $P_{Q} \neq 0$. (Note that Lemma 1 implies $Q>P$.) Further $P_{Q}$ $=P_{Q}^{2}$ so there is a $\Pi \in P_{Q}$ such that $\Pi \neq 0, P_{Q} \nsubseteq 0$ : $\Pi$. But Lemma 1 , together with the choice of $Q$, guarantees that $0: \Pi$ is $Q_{Q}$-primary. Hence $P_{Q}$ $\subset 0$ : $\Pi$ and we have a contradiction. In order to show that, for all $\Pi \in P$, $\Pi \in \Pi P$ we note that the first portion of this proof shows that $P+0: \Pi=R$. Hence $1=p+b$ where $p \in P$ and $b \in 0$ : $\Pi$. Thus $\Pi=\Pi p \in \Pi P$.

TheOREM $3\left({ }^{2}\right)$. Let $R$ be a u-ring in which the set of proper prime ideals is inductive. Then for all $\Pi \in R, \Pi \in \Pi R$. If, in addition, the zero ideal of $R$ is a finite product of prime ideals, then $R$ has an identity element.

Proof. Let $R^{*}$ be a ring of characteristic 0 obtained by adjoining an identity to $R$. Then $R^{*} / R \cong Z$ so that $R$ is prime in $R^{*}$ and the descending chain condition for prime ideals holds in $R^{*} / R$. Further, if $\left\{P_{\alpha}\right\}$ is a chain of prime ideals of $R^{*}$ such that $R \subset \cup P_{\alpha}$, then $\left\{P_{\alpha} \cap R\right\}$ is a chain of prime ideals of $R$ such that $R=\bigcup\left(P_{\alpha} \cap R\right)$. By hypothesis $R=P_{\alpha} \cap R \subset P_{\alpha}$ for some $\alpha$ then. Thus, the first statement holds in view of Lemma 2 .

To prove the second statement, we note that if $P$ is a prime ideal of $R$, then $R / P$ is a $u$-domain satisfying the hypothesis of Theorem 3 and therefore contains an identity element. Our second statement then follows from the following observation, which is easily established: if $A, B$ are ideals of a commutative ring $S$ and if $S / A$ and $S / B$ are rings with identity, then $S / A B$ is a ring with identity.

We shall need to apply Theorem 3 in a very special case, namely when

$\left({ }^{2}\right)$ We are indebted to the referee for Theorem 3 , which generalizes the following result, sufficient for the application of this paper of ours: If $R$ is a finite dimensional $u$-ring and $\Pi \in R$, then $\Pi \in \Pi R$. 
$R$ is a $u$-ring of dimension one or zero. Our first use of Theorem 3 is in proving

TheOREM 4. Let $R$ be a commutative ring. Then $R$ satisfies (*) if and only if every ideal of $R$ is equal to its kernel.

Proof. Suppose every ideal of $R$ is equal to its kernel. If $A$ is an ideal of $R$ such that $\sqrt{ } A=P$ is a prime ideal, then $P$ is the unique minimal prime ideal of $A$. Thus if $Q$ is the isolated component of $A$ belonging to $P$, then the kernel of $A$ is $Q$. Since $A$ is equal to its kernel, $A=Q$ and $A$ is $P$-primary.

Suppose now that $R$ satisfies $\left({ }^{*}\right)$. If $R$ is a primary domain or a ring in which every element of $R$ is nilpotent, then every ideal of $R$ is primary so that every ideal of $R$ is equal to its kernel. If $R$ is a $u$-domain, then to show a proper ideal $A$ is equal to its kernel $A^{*}$, it suffices by Theorem 3 , to show that if $x \in A^{*}$, then $R(x) \subset A$ or, equivalently, $A:(x)=R$. Since $R$ is a $u$-domain, it is even sufficient to show $A:(x)$ is contained in no proper prime ideal of $R$. We know that $A:(x)$ is contained in no minimal prime ideal of $A[10$, Theorem 9, p. 738], and also that $A<A:(x)$. To obtain the desired conclusion then we need only show that if $M$ is a maximal ideal of $R$ containing a prime ideal $P$ such that $M>P \supset A$, then $A:(x) \nsubseteq M$. This follows because $x \in A^{*} \subset P$ so that by Theorem $1\left(\mathrm{~d}^{\prime}\right) 0=x m$ for some $m \in R-M$. Consequently, $m \in A:(x)-M$. This completes the proof.

Theorem 5. A u-ring $R$ satisfies $\left(^{*}\right)$ if and only if for $p \in P$, a nonmaximal proper prime ideal of $R, p \in p P$.

Proof. We first show that a $u$-ring $R$ satisfying $\left({ }^{*}\right)$ has the property described. Thus if $M$ is a maximal ideal containing $P$, Theorem 1 (d) implies $p=m p$ for some $m \in M$. If $r \in R-M$, then we have $(r-r m) p=0$. Thus if $A$ is the annihilator of $p, A \subset M$. This implies $P+A$ has no maximal ideal divisors. Then by Theorem 2 (a), $P+A=R$. Therefore $m=q+a$ where $q \in P, a \in A$. Then $p=m p=q p+a p=q p$ and $p \in p P$ as asserted.

Conversely, if $p \in p P$ whenever $p$ is an element of the nonmaximal prime ideal $P$, then to show $R$ satisfies $\left(^{*}\right)$ we need only show that $R$ has dimension less than or equal to one by Theorem 1 . We suppose there exists a chain $P<Q<M<R$ of prime ideals of $R$. If $q \in Q-P$, then by hypothesis $q=s q$ for some $s \in Q$. If then $t \in R-Q,(t-t s) q=0$ so that $(t-t s) \in P$ $\subset Q$ and $t \in Q$ since $t s \in Q$. This contradiction shows that $R$ has dimension $\leqq 1$.

Theorem 5 shows that if $A$ is an ideal of a $u$-ring $R$ satisfying (*) such that $A$ is contained in a nonmaximal prime ideal $P$, then $A=P A$. This is the first step in the fulfillment of condition (F) in $u$-rings satisfying $\left({ }^{*}\right)$. More generally, we have

Theorem 6. If the u-ring $R$ satisfies $\left({ }^{*}\right)$, if $A, B$, and $P$ are ideals of $R, P$ a prime ideal, such that $A<P<B$, then $A=B A$. 
Proof. If $P$ is maximal, $B=R$ and the conclusion follows from Theorem 3 . If $P$ is nonmaximal, Theorem 5 shows that Theorem 6 holds.

\section{Rings in which condition ( $F$ ) holds.}

Definition. $A$ ring $R$ is said to satisfy condition (F) if whenever $A$ and $P$ are ideals of $R$ such that $P$ is prime and $A<P$, then there exists an ideal $B$ of $R$ such that $A=P B$. (Condition (F) has been considered by Asano in $[2$, Theorem 2, p. 85] and by Mott in [19].)

Theorem 7. Suppose $D$ is an integral domain in which condition (F) holds. If $D=D^{2}, D$ is a Dedekind domain. If $D \neq D^{2}$, then every nonzero ideal of $D$ is a power of $D$. (By [4], $D$ is the unique maximal ideal of a rank one discrete valuation ring with residue field $G F(p)$ for some prime integer $p$.)

Proof. We first show that $D$ is Noetherian. To do this, it suffices to show that if $P$ is a prime ideal of $D$, then $P$ has a finite basis [3, Theorem 2 , p. 29]. This we proceed to do. If $P$ is principal, $P$ has a finite basis. If $P$ is not principal, and if $p$ is a nonzero element of $P$, then $(p)<P$ so that $(p)=P A$ for some ideal $A$ of $D$. Therefore $P$ is invertible, and consequently, $P$ has a finite basis.

If then $D=D^{2}, D$ is an integral domain with unit [2, Lemma 3, p. 86 ], and is therefore a Dedekind domain [20, p. 234].

If $D \neq D^{2}$ and $A$ is a nonzero proper ideal of $D$, we wish to show $A$ is a power of $D$. Because $D$ is Noetherian, it suffices to prove this under the assumption that every proper divisor of $A$ in $D$ is a power of $D$. By hypothesis, there exists an ideal $B$ of $D$ such that $A=D B$. Because $D$ is a Noetherian integral domain without unit, $B>A$ so that $B=D^{k}$ for some integer $k$. Therefore $A=D^{k+1}$ and our proof is complete.

From Theorem 7 , we immediately obtain:

Corollary 1. If $P$ is a prime ideal of a ring $R$ satisfying condition (F), then every ideal of $R / P$ may be expressed as a product of prime ideals.

Theorem 8. If $P$ is a prime ideal of a ring $R$ satisfying condition $(\mathrm{F})$, if $B \subset P$, and if $A$ is a proper divisor of $P$ in $R$, then $B=A B$ and hence $B \subset \bigcap_{n=1}^{\infty} A^{n}$. In particular, $B \subset \bigcap_{n=1}^{\infty} R^{n}$.

Proof. We first show that $P=P A$. Thus if $M$ is a prime ideal properly containing $P$, then $P=M C$ for some ideal $C$ of $R$. Since $P$ is prime and $P<M, C \subset P \subset C, C=P$, and $P=M P$. Now $A / P$ is a nonzero ideal of the domain $R / P$. By Corollary $1, A / P=\prod_{i=1}^{t}\left(P_{i} / P\right)=\left[\left(\prod_{i=1}^{t} P_{i}\right)+P\right] / P$ for some collection $\left\{P_{1}, P_{2}, \cdots, P_{t}\right\}$ of prime ideals properly containing $P$. Hence $A=\left(\coprod{ }_{i=1}^{t} P_{i}\right)+P$ and $A P=\left(\coprod_{i=1}^{t} P_{i}\right) P+P^{2}=P+P^{2}=P$ by what we have just shown previously.

Now if $B<P, B=P D$ for some ideal $D$ of $R$ so that $B=(A P) D=A(P D)$ 
$=A B$. It follows that for $B \subset P, B=A B=A^{2} B=\cdots$, and consequently, $B \subset \bigcap_{n=1}^{\infty} A^{n}$.

Lemma 3. If $A$ is an ideal of a ring $S$ such that there are no ideals properly between $A$ and $A^{2}$, then the only ideals between $A$ and $A^{n}$ are $A, A^{2}, \cdots, A^{n}$.

Proof. Without loss of generality we may assume $A^{n}=(0)$. If $A>A^{2}$, it is well known that for a positive integer $i$, there are no ideals properly between $A^{i}$ and $A^{i+1}$. Hence if $x \in A^{i}-A^{i+1}$, then $A^{i}=A^{i+1}+(x)$ so that in $R /(x), A^{i} /(x)=[A /(x)]^{i}=[A /(x)]^{i+1}=\cdots=[A /(x)]^{n}=A^{n}+(x) /(x)$ $=(x) /(x)$. Hence $A^{i}=(x)$. This implies Lemma 3 .

Theorem 9. If a ring $R$ satisfies condition $(\mathrm{F})$, then $R$ satisfies $\left({ }^{*}\right)$.

Proof. We suppose $Q$ is an ideal of $R$ such that $\sqrt{ } Q=P$ is prime. If $P=R$, $Q$ is primary. If $P<R$, we suppose $Q$ is not primary so that for some $p \in P-Q, r \in R-P, p r \in Q$. We let $A=Q+(p) P$. Then $p \notin A$ since otherwise we would have $p \equiv q p(Q)$ for some $q \in P$. Hence $p \equiv q p \equiv q^{2} p \equiv$ $\ldots \equiv q^{n} p \equiv 0(Q)$ for some integer $n$, contrary to the assumption that $p \notin Q$. Hence $p \notin A$, but then by Theorem $8, A+(p)=[A+(p)][P+(r)] \subset$ $A+(p) P+(p r) \subset A$, a contradiction. Hence $Q$ is primary for $P$.

THEOREM 10. If $R$ is a nonidempotent ring satisfying condition (F), then every nonzero ideal of $R$ is a power of $R$.

Proof. If $r \in R-R^{2}$, then there is no ideal $A$ of $R$ such that $(r)=R A$ so that $(r) \varangle R$; that is, $R=(r)$ and there are no ideals properly between $R$ and $R^{2}$.

If $r$ is nilpotent, then $R^{k}=(0)$ for some integer $k$. If $B$ is an ideal of $R$, then $R^{k} \subset B \subset R$, and Lemma 3 implies $B$ is a power of $R$.

If $r$ is not nilpotent, then from Theorems 1,2 , and 9 , we see that $R$ is a domain. The desired conclusion then follows from Theorem 7 .

Theorem 10 determines the structure of nonidempotent rings satisfying condition (F). If $R$ is an idempotent ring satisfying condition (F) and if $r$ is a nonzero element of $R,(r)=R(r)$ so that $r=x r$ for some $x \in R$. Because $r \neq 0, x$ is not nilpotent and if $R$ is a domain, $x$ is even an identity element of $R$. In either case, Theorem 1 implies $R$ is a $u$-ring. By Theorem $3, R A=A$ for each ideal $A$ of $R$. This implies that if $A$ and $B$ are relatively prime ideals of $R$, then $A \cap B=[A \cap B] R=[A \cap B][A+B]=A(A \cap B)$ $+B(A \cap B) \subset A B \subset A \cap B$ so that $A \cap B=A B$. These remarks will be useful as we consider idempotent rings satisfying condition (F). We first determine some additional properties of idempotent rings satisfying condition (F). Lemmas 4 and 5 and Theorem 11 represent generalizations of Mott's results in [19] to the case of a ring without identity.

Lemma 4. If $R$ is an idempotent ring satisfying condition (F), if $P$ is a 
prime ideal of $R$, and if $A$ is an ideal of $R$ such that $A \subset P^{n}$ for some nonnegative integer $n$, then there exists an ideal $B$ of $R$ such that $A=P^{n} B$.

Proof. We use induction on $n$, the conclusion being true for $n=1$ and $n=0$ by hypothesis and by our earlier observation that $C=R C$ for each ideal $C$ of $R$. If the conclusion holds for $n=k$ and if $A \subset P^{k+1}$, then $A=B P^{k}$ for some ideal $B$. If $P^{k}=P^{k+1}$ then $A=B P^{k+1}$. If $P^{k+1}<P^{k}$, then $P^{k+1}$ is $P$-primary because $R$ is a $u$-ring satisfying. ( $\left.{ }^{*}\right)$. Since $B P^{k} \subset P^{k+1}$ and $P^{k}$ $\llbracket P^{k+1}, B \subset P$ and $B=P C$ for some ideal $C$ of $R$. Thus $A=P^{k+1} C$.

Lemma 5. If $M$ is a maximal ideal of the idempotent ring $R$ satisfying condition (F), and if the powers of $M$ properly descend, then $P=\bigcap_{n=1}^{\infty} M^{n}$ is a prime ideal.

Proof. If $x, y \in R-P$, then we may choose integers $k$ and $m$ such that $x \in M^{k}-M^{k+1}, y \in M^{m}-M^{m+1}$. By Lemma 4 , there exists ideals $B$ and $C$ of $R$, neither of which is contained in $M$, such that $(x)=M^{k} B$ and $(y)=M^{m} C$. Now $(x y)=M^{m+k} B C, M^{m+k} \nsubseteq M^{m+k+1}$ and $B C \nsubseteq M$. Thus $x y$ is not contained in the primary ideal $M^{m+k+1}$ and hence is not in $P$. This proves that $P$ is a prime ideal.

Theorem 11. If $Q$ is a primary ideal of the idempotent ring $R$ satisfying condition (F), then $Q$ is a power of its radical.

Proof. We let $P=\sqrt{ } Q$. If $P=R$, then $Q=P$ because $R$ is a $u$-ring. If $P$ is a nonmaximal proper ideal, then $Q=P$ by Theorem 1 . If $P$ is a maximal ideal of $R$, we consider two cases:

Case 1. If $Q \subset \bigcap_{n=1}^{\infty} P^{n}$, then Lemma 5 implies that $P^{n}=P^{n+1}$ for some positive integer $n$. If then $x \in P^{n}$, Lemma 4 implies $(x)=P^{n} B=P^{2 n} B$ $=P^{n}(x)$ so that $x=t x$ for some $t \in P^{n}$. Since $t^{r} \in Q$ for some integer $r$, $x=t^{r} x \in Q$ also, and $Q=P^{n}$.

Case 2. There exists an integer $n$ such that $Q \subset P^{n}, Q \nsubseteq P^{n+1}$. Then $Q=P^{n} C$ for some ideal $C \nsubseteq P$ by Lemma 4 . Since $Q$ is primary for $P, P^{n}$ $\subset Q \subset P^{n}$ and $Q=P^{n}$.

TheOREM 12. Let $R$ be a ring satisfying condition (F). In $R$ the following conditions hold:

(i) $R$ satisfies $\left(^{*}\right)$,

(ii) every primary ideal of $R$ is a prime power, and

(iii) if $P$ is a proper prime ideal of $R$ and if the ideal $A$ and the positive integer $n$ are such that $A \subset P^{n}, A \nsubseteq P^{n+1}$, then there exists an element $y \in$ $R-P$ such that $P^{n}=A:(y)$.

(Condition (iii) resembles what Krull calls " $Q u$-Bedingung für i.K.I." in [12].)

Proof. Properties (i) and (ii) hold in $R$ by Theorems 9, 10 and 11. Property 
(iii) is trivially true by Theorem 10 if $R \neq R^{2}$. We proceed to show (iii) holds if $R=R^{2}$.

Since $P^{n} \neq P^{n+1}, P$ is a maximal ideal. By Lemma $4, A=P^{n} B$ for some ideal $B$ such that $B \nsubseteq P$. Now $P^{n-1} \nsubseteq P^{n}$ and $P^{n}$ is $P$-primary. Therefore $P^{n-1} B \nsubseteq P^{n}$ and in particular, $P^{n-1} B>A$. If $C$ is the ideal generated by the set $B-(P \cap B)$, then clearly $B=(P \cap B)+C$. But $P$ is maximal and $B \nsubseteq P$ so that $P \cap B=P B$. Hence $B=P B+C$ and we have $A$ $<P^{n-1}(P B+C)=P^{n} B+P^{n-1} C=A+P^{n-1} C$. This implies there exists $y \in B-P$ such that $P^{n-1} y \nsubseteq A$. Since $y \in B-P, P^{n} \subset A:(y) \subset P$. It follows that $A:(y)$ has radical $P$ and is therefore a power of $P$ by (i) and (ii). But by choice of $y, P^{n-1} \nsubseteq A:(y)$. Consequently, $P^{n}=A:(y)$.

Corollary 2. If $P$ is a prime ideal of a ring $R$ satisfying condition (F), there are no ideals properly between $P$ and $P^{2}$.

Proof. Suppose $P^{2} \subset A \subset P$. Then $A$ has radical $P$ and is therefore primary for $P$. Consequently, $A$ is a power of $P$ so that $A=P$ or $A=P^{2}$.

Theorem 13. A ring $S$ in which conditions (i), (ii), and (iii) of Theorem 12 hold is an AM-ring.

Proof. Since (i) is valid in $S$, Theorem 1 applies. Thus if $S$ is not a $u$-ring, Theorem 1 implies each nonzero ideal of $S$ has radical $S$, is therefore primary for $S$, and hence is a power of $S$.

If $S$ is a $u$-ring, then Theorem 1 implies $S$ has dimension less than two and the only ideal primary for a nonmaximal prime ideal $P$ of $S$ is $P$ itself. Further, $S A=A$ for each ideal $A$ of $S$ by Theorem 3. To show then that $S$ is an $A M$-ring, it suffices to show that if $A$ and $B$ are ideals of $S$ such that (0) $<A<B<S$, then $A=B C$ for some ideal $C$ of $S$. Because (i) holds in $S$, every ideal of $S$ is equal to its kernel. Consequently, it suffices to produce an ideal $C$ of $S$ such that $A$ and $B C$ have the same kernels. This we proceed to do.

If $\left\{P_{\alpha}\right\}_{\alpha \in T}$ is the collection of minimal prime ideals of $A$, then by (ii) for each $\alpha \in T$ we may choose integers $n_{\alpha}$ and $m_{\alpha}$ such that (1) $P_{\alpha}^{n_{\alpha}}$ is the isolated primary component of $B$ belonging to $P_{\alpha}$ if $P_{\alpha} \supset B$, and $n_{\alpha}=0$ if $P_{\alpha} D B$, (2) $P_{\alpha}^{m_{\alpha}}$ is the isolated primary component of $A$ belonging to $P_{\alpha}$, and (3) $m_{\alpha} \geqq n_{\alpha}$ for $\alpha \in T$. We set $C=\bigcap_{\alpha \in T} P_{\alpha}^{m_{\alpha}-n_{\alpha}}$. It is then clear that $B C \subset A \subset C$. Using this and the fact that $A<B$, we can show that $\left\{P_{\alpha}\right\}$ is the set of minimal primes of $B C$. Hence for each $\alpha \in T$, we may choose an integer $t_{\alpha} \geqq m_{\alpha}$ such that $P_{\alpha}^{t_{\alpha}}$ is the isolated primary component of $B C$ belonging to $P_{\alpha}$. To complete the proof we need only show that $P_{\alpha}^{m_{\alpha}}=P_{\alpha}^{t_{\alpha}}$ for each $\alpha \in T$.

If $P_{\alpha}^{m_{\alpha}}=P_{\alpha}^{m_{\alpha}+1}$ it is clear that $P_{\alpha}^{m_{\alpha}}=P_{\alpha}^{t_{\alpha}}$. If $P_{\alpha}^{m_{\alpha}} \neq P_{\alpha}^{m_{\alpha}+1}$ then $P_{\alpha}$ is maximal because of (i). By (iii), there exists $y \in S-P_{\alpha}$ such that $P_{\alpha}^{m_{\alpha}}=A:(y)$. If $\beta \in T, \beta \neq \alpha$, then we have $P_{\alpha}^{m_{\alpha}}(y) \subset P_{\beta}^{m_{\beta}}, P_{\alpha}^{m_{\alpha}} \Subset P_{\beta}$ so that $y \in P_{\beta}^{m_{\beta}}$. 
Hence $y \in \bigcap_{\beta \neq \alpha} P_{\beta}^{m_{\beta}}=A^{\prime} \subset B^{\prime}=\bigcap_{\beta \neq \alpha} P_{\beta}^{n_{\beta}}$ and $A^{\prime} \subset C^{\prime}=\bigcap_{\beta \neq \alpha} P_{\beta}^{m_{\beta}-n_{\beta}}$, but $y \notin P_{\alpha}$. This implies $B=P_{\alpha}^{n_{\alpha}} B^{\prime}$ (if $P_{\alpha} D B, P_{\alpha}^{n_{\alpha}}=S, B^{\prime}=B$, and $B$ $=P_{\alpha}^{n_{\alpha}} B^{\prime}$, while if $P_{\alpha} \supset B$, then $P_{\alpha}^{n_{\alpha}}$ and $B^{\prime}$ are relatively prime since $P_{\alpha}$ is maximal, and consequently $P_{\alpha}^{n_{\alpha}} B^{\prime}=P_{\alpha}^{n_{\alpha}} \cap B^{\prime}=B$ ). Similarly, $C$ $=P_{\alpha}^{m_{\alpha}-n_{\alpha}} C^{\prime}$. Therefore $B C=P_{\alpha}^{m_{\alpha}} B^{\prime} C^{\prime}$ where $B^{\prime} C^{\prime} \subset P_{\alpha}$. It follows that $B C \subset P_{\alpha}^{m_{\alpha}}, B C \nsubseteq P_{\alpha}^{m_{\alpha}+1}$ so that $P_{\alpha}^{m_{\alpha}}=P_{\alpha}^{t_{\alpha}}$ as we wished to show.

REMARK. Condition (iii) of Theorem 12 is equivalent, in rings satisfying (i) and (ii), to the following condition:

(iv) If $P$ is a minimal prime ideal of an ideal $B$, if $P^{n}$ is the isolated primary component of $B$ belonging to $P$, and if $P^{n} \neq P^{n+1}$, then $P$ does not contain the intersection of the remaining isolated primary components of $B$.

Thus, a ring $R$ is an $A M$-ring if and only if (i), (ii), and (iv) hold in $R$. For rings with unit, this result was proved by Mott in [19].

Condition (i) and (ii) of Theorem 12 may be replaced by a single condition in the case of a $u$-ring. The proof of the following theorem uses extensively the results of [21, pp. 221-228].

Theorem 14. Condition (i) and (ii) of Theorem 12 hold in the u-ring $S$ if and only if given a proper prime ideal $P$ of $S, S_{P}$ is a Dedekind domain or a special primary ring.

Proof. We first suppose (i) and (ii) hold in $S$ and that $P$ is a proper prime ideal of $S$. If $T=S-P$, if $A_{P}$ is the set of elements $x$ of $S$ such that $x t=0$ for some $t \in T$, and if $\bar{T}$ is the set of residue classes $\bar{t}$ of $\bar{S}=S / A_{P}$, where $t \in T$, then by definition, $S_{P}=\bar{S}_{\bar{T}}=\bar{S}_{\bar{P}}$. If $P$ is nonmaximal, then Theorem 1 implies $A_{P}=P$ and $S_{P}$ is a field. If $P$ is maximal and properly contains a nonmaximal prime ideal $P^{\prime}$, then $A_{P}=P^{\prime}$ by Theorem 1 so that $S_{P}$ is a onedimensional integral domain with unit. Every nonzero proper ideal is therefore primary for the unique maximal ideal of $S_{P}$. But we can easily see that (ii) holds in $S_{P}$ whenever it holds in $S$. Consequently, every nonzero proper ideal of $S_{P}$ is a power of the unique maximal ideal of $S_{P}$. It follows that $S_{P}$ is a Dedekind domain. Finally, if $P$ is maximal and minimal, then $A_{P}$ is $P$-primary. From (ii), $A_{P}=P^{n}$ for some integer $n$. This implies that $S_{P}$ $\cong S / P^{n}$, so that $S_{P}$ is a primary ring in which every primary ideal is a power of its radical; that is, $S_{P}$ is a special primary ring.

Conversely, if each $S_{P}$ is a Dedekind domain or a special primary ring, if $P$ is a nonmaximal proper prime ideal of $S$, and if $M$ is a maximal ideal containing $P$, then $S_{M}$ has dimension greater than zero and is therefore a Dedekind domain. This implies $S$ has dimension less than two. Moreover, $P=A_{M}$ so that $S$ satisfies (*) by Theorem $1\left(\mathrm{~d}^{\prime}\right)$. To prove (ii) it suffices, in view of Theorem $1\left(\mathrm{~d}^{\prime \prime}\right)$, to prove that if $Q$ is primary for the maximal ideal $M, Q$ is a power of $M$. This follows immediately from the fact that powers of $M$ are $M$-primary and that every primary ideal of $S_{M}$ is a prime power. 
We proceed to find another equivalence to (i) and (ii) of Theorem 12 in $u$-rings, but first we need the following lemma.

Lemma 6. If $D$ is a domain in which every ideal with prime radical is a prime power, then nonzero proper prime ideals are maximal.

Proof. Let $P_{1}$ be a nonzero proper prime ideal of $D$ and suppose $p \in P_{1}$. Then if $P$ is a minimal prime of $(p)$, let $Q_{i}$ be the isolated $P$-primary component of $\left(p^{i}\right)$. Clearly $Q_{i} \supset Q_{i+1}$ for each integer $i$. In fact, $Q_{i}>Q_{i+1}$ because $p^{i} \in Q_{i}$ and $p^{i} \in Q_{i+1}$. Since every ideal with prime radical is a prime power, we see that $Q_{i}=P^{n_{i}}$ where $n_{i}>n_{i+1}$ for each $i$. Therefore, there is an integer $k>1$ such that $P^{k}$ is $P$-primary and $P^{k-1}>P^{k}$. Suppose $y \in$ $P^{k-1}-P^{k}$ and $a \in D-P$. Since $P^{k}$ is $P$-primary, ay $\notin P^{k}$ so that $P^{k}<$ $P^{k}+(a y) \subset P^{k-1}$. Since the radical of $P^{k}+(a y)$ is $P$, we see that $P^{k}+$ $(a y)=P^{k-1}$. Thus $y \in P^{k}+(a y)$ and, as a consequence, $y=q+$ ray + nay where $q \in P^{k}, r \in D$ and $n$ is an integer. If $d \in D$, to prove $P$ is a maximal ideal, it suffices to show $d \in P+(a)$. It follows that $d y=d q+d r a y+d n a y$ so that $y(d-d r a-d n a)=d q \in P^{k}$. Since $P^{k}$ is $P$-primary and $y \notin P^{k}$ it follows that $d-d r a-d n a \in P$ and consequently $d \in P+(a)$. Hence $P$ is maximal. Since $P_{1} \supset P, P_{1}=P$, and $P_{1}$ is maximal.

Theorem 15. Conditions (i) and (ii) of Theorem 12 hold in the u-ring $S$ if and only if every ideal with prime radical is a prime power.

Proof. Clearly (i) and (ii) imply that every ideal with prime radical is a prime power. Suppose then that every ideal with prime radical is a prime power. We wish to show that $S$ satisfies $\left({ }^{*}\right)$. By Lemma 6 , it follows that the dimension of $S$ is less than two. If $S$ is zero-dimensional, then $S$ satisfies $\left({ }^{*}\right)$ by Theorem 1 . It suffices to show $S$ satisfies $\left({ }^{*}\right)$ under the assumption that $S$ is one-dimensional. If $S$ is a domain, then by Theorem $2, S$ contains an identity and hence powers of a maximal ideal are primary so that in this case $S$ satisfies $\left({ }^{*}\right)$. Assume then that nonmaximal prime ideals of $S$ are nonzero. It suffices by Theorem 14 , to show that $S_{P}$ is either a Dedekind domain or a special primary ring for each proper prime ideal $P$ of $S$. We note that every ideal of $S_{P}$ with prime radical is a prime power, since this is true in $S$. In particular, if $P$ is a minimal prime ideal of $S$, then $S_{P}$ is a ring with only one prime ideal and every ideal of $S_{P}$ is a power of this prime ideal; that is, $S_{P}$ is a special primary ring. If $P$ is not minimal, let $P^{\prime}$ be a minimal prime contained in $P$. It is straightforward to show that $P^{\prime}=\bigcap_{n=1}^{\infty} P^{n}$ by considering the one-dimensional $u$-domain $S / P^{\prime}$. Thus $P^{\prime}$ is the unique prime ideal properly contained in $P$, and $S_{P}$ is a onedimensional $u$-ring containing only two prime ideals. Consequently, every ideal of $S_{P}$ has prime radical and hence, is a power of a prime ideal. It follows from $[2$, Theorem 1, p. 83$]$ that $S_{P}$ is a finite direct sum of Dedekind domains and special primary rings. Since $S_{P}$ is one-dimensional and contains a unique 
maximal ideal, it follows that $S_{P}$ is a Dedekind domain. The proof of the theorem is complete.

REMARK. If $B$ is an idempotent ideal of an $A M$-ring $R$ and if $A \subset B$, then $A=A B$ because $B=B^{2}$ and if $A<B$, then $A=B C=B^{2} C=B A$. In particular, an idempotent $A M$-ring is a multiplication ring.

By Theorem 10 , if $R$ is a nonidempotent $A M$-ring, then the only idempotent ideal of $R$ is (0). Thus an $A M$-ring containing a nonzero idempotent ideal is an idempotent ring and hence is a multiplication ring.

Based on our previous results, we are now able to give simple proofs to two theorems of Mori which give characterizations of idempotent multiplication rings in two important special cases. First we need the following lemma.

Lemma 7. If $A$ and $B$ are ideals of an $A M$-ring $R$ such that $A<B$ and $B$ is idempotent, then there exists an idempotent element $b \in B-A$. Therefore, in an $A M$-ring idempotent ideals are generated by idempotent elements.

Proof. If $y \in B-A$, then $(y)=(y) B$ so that $y=x y$ for some $x \in B$. Since $y \notin A$, no power of $x$ is in $A$. Thus if $C$ is the ideal of nilpotent elements of $B$, then $C<B$. We let $M=[C:(x)] \Gamma_{1} B$ and $N=[C: M] \cap B$. If $m=r x+t x \in M \cap(x)$, where $t$ is an integer, then $m^{2} \in C$ so that $m \in C$. Similarly, if $m=n \in M \cap N$, then $n^{2} \in C$ and again $n \in C$. Therefore, $M \cap(x) \subset C=M \cap N$.

For some ideal $D$ of $R,(x)=D[C+(x)+M]=D B[C+(x)+M]$. Now $D B M \subset(x) \cap M \subset C$ so that $D B \subset N$. Thus $(x) \subset C+N(x)$ or $x \equiv n x(C)$ for some $n \in N$. This implies $\left(n-n^{2}\right) x \in C$ so that $n-n^{2} \in N$ $\cap M=C$. Consequently $\left(n-n^{2}\right)^{k}=0$ for some $k$ or $n^{k}=v n^{2 k}$. It follows that $v n^{k}$ is an idempotent element of $B$. If $v n^{k} \in A$, then $n^{k} \in A$ also. Since $x \equiv n^{k} x(C)$, we would then have $x \in A+C$ so that for some integer $r$, $x^{r} \in A$. This is contrary to what we observed earlier about $x$. Thus $v n^{k} \notin A$.

We now prove a theorem due to Mori [18, Theorem 8, p. 9].

Theorem 16. An indecomposable multiplication ring $R$ with identity is either a Dedekind domain or a special primary ring.

Proof. Because $R$ is indecomposable, 0 and 1 are the only idempotent elements of $R$. By Lemma 7, (0) and $R$ are the only idempotent ideals of $R$. Suppose $P$ is a maximal ideal of $R$. If the powers of $P$ properly descend, then Lemma 5 implies $Q=\bigcap_{n=1}^{\infty} P^{n}$ is a nonmaximal prime ideal of $R$. Since $R$ satisfies $\left(^{*}\right), Q=Q^{2}$ so that $Q=(0)$. It follows that $R$ is an integral domain with unit satisfying condition $(F)$ and therefore is a Dedekind domain by Theorem 7 . If the powers of $P$ do not properly descend, then for some integer $n>0, P^{n}=P^{2 n}=(0)$. This implies $P$ is the only proper prime ideal of $R$ and if $A$ is a proper ideal of $R$, then $P \supset A \supset P^{n}$. By Lemma 
3 and Corollary $2, A$ is a power of $P$. Therefore, $R$ is a special primary ring. The following theorem is also due to Mori [18, Theorem 9, p. 9 ].

Theorem 17. Suppose $R$ is an idempotent AM-ring in which every idempotent element may be written as a finite sum of primitive orthogonal idempotents. Then $R$ is a direct sum of Dedekind domains and special primary rings.

Proof. By Lemma 7, $R$ contains a nonzero idempotent element. If $e \neq 0$ is a primitive idempotent of $R$, then $(e)$ is a direct summand of $R$ and hence a homomorphic image of $R$. Because $e$ is primitive, $(e)$ is an indecomposable $A M$-ring with unit, and therefore is a Dedekind domain or a special primary ring by Theorem 16. Hence $(0)$ is the only idempotent proper ideal of $(e)$.

Now suppose $A$ is any idempotent ideal of $R$ not containing $(e)$. If then $B=(e) \cap A$, then $B=B(e)$ and $B=B A$ because $(e)$ and $A$ are idempotent. It follows that $B=B(e)=B A(e) \subset B[A \cap(e)]=B^{2}$ so that $B=B^{2}$. As we have just seen, this implies $B=(0)$ and $A+(e)=A \oplus(e)$. This implies that if $S$ is an ideal of $R$ maximal with respect to the property that $S$ is a direct sum of Dedekind domains and special primary rings, then $S$ contains every primitive idempotent of $R$ and hence every idempotent of $R$. By Lemma $7, S=R$. This completes the proof of the theorem.

The converse of Theorem 17 is contained in the following result. $A$ straightforward proof can be given and will be omitted.

Theorem 18. Suppose $\left\{R_{\alpha}\right\}_{\alpha \in T}$ is a collection of idempotent AM-rings and $R=\sum \oplus R_{\alpha}$. Then $R$ is again an idempotent AM-ring. If each $R_{\alpha}$ is indecomposable, then every idempotent element of $R$ is a finite sum of primitive orthogonal idempotents.

An idempotent Noetherian ring contains an identity $e$ and $e$ is a finite sum of primitive orthogonal idempotents. In view of this fact, we obtain:

Corollary 3. A Noetherian ring $R$ is a multiplication ring if and only if $R$ is a finite direct sum of Dedekind domains and special primary rings.

\section{REFERENCES}

1. Y. Akizuki, Bemerkungen über den Aufbau des Nullideals, Proc. Phys.-Math. Soc. Japan 14 (1932), 253-262.

2. K. Asano, Über kommutative Ringe, in denen jedes Ideals als Produkt von Primidealen darstellenbar ist, J. Math. Soc. Japan 1 (1951), 82-90. $27-42$.

3. I. S. Cohen, Commutative rings with restricted minimum condition, Duke Math. J. 17 (1950),

4. Robert W. Gilmer, Jr., A classical theorem of Noether in ideal theory, Pacific J. Math. 13 (1963), 579-583.

5. Commutative rings containing at most two prime ideals, Michigan Math. J. 10 (1963), 263-268.

6. __ Eleven nonequivalent conditions on a commutative ring (submitted for publication).

7. __ Extensions of results concerning rings in which semi-primary ideals are primary, Duke Math. J. 31 (1964), 73-78. 
8. _ Rings in which semi-primary ideals are primary, Pacific J. Math. 12 (1962), 1273-1276.

9. W. Krull, Idealtheorie, Chelsea, New York, 1948.

10. __ Idealtheorie in Ringen ohne Enclichkeitsbedingung, Math. Ann. 101 (1929), 729-744.

11. _ Über allgemeine Multiplikationsringe, Tôhoku Math. J. 41 (1936), 320-326.

12. __ Über Laskershe Ringe, Rend. Circ. Mat. Palermo (2) 7 (1958), 155-166.

13. __ Über Multiplikationsringe, S.-B. Heidelberger Akad. Wiss. 5 (1925), 13-18.

14. S. Mori, Axiomatische Begrundung des Multiplikationsringe, J. Sci. Hiroshima Univ. Ser. A 3 (1932), 45-59.

15. , Über allgemeine Multiplikationsringe. I, J. Sci. Hiroshima Univ. Ser. A. 4 (1934), 1-26.

16. ¿Über allgemeine Multiplikationsringe.II, J. Sci. Hiroshima Univ. Ser. A 4 (1934), 99-109.

17. _ـ Über Idealtheorie der Multiplikationsringe, J. Sci. Hiroshima Univ. Ser. A 19 (1956), 429-437.

18. __ Struktur der Multiplikationsringe, J. Sci. Hiroshima Univ. Ser. A 16 (1952), 1-11.

19. Joe Leonard Mott, Equivalent conditions for a ring to be a multiplication ring, Canad. J. Math. 16 (1964), 429-434.

20. N. Nakano, Über die Umkehrbarkeit der Ideale im Integritatsbereiche, Proc. Imp. Acad. Tokyo 19 (1943), 230-234.

21. O. Zariski and P. Samuel, Commutative algebra, Vol. I, Van Nostrand, Princeton, N. J., 1958.

\author{
Florida State University, \\ Tallahassee, Florida \\ Louisiana State University, \\ Baton Rouge, Louisiana
}

